\title{
Prediction of Partition Coefficients of Environmental Toxins Using Computational Chemistry Methods
}

\author{
David van der Spoel, ${ }^{*}+\oplus$ Sergio Manzetti, ${ }^{\dagger+}+\odot$ Haiyang Zhang, ${ }^{\S}$ and Andreas Klamt ${ }^{\|, \perp}$
}

${ }^{\dagger}$ Uppsala Center for Computational Chemistry, Science for Life Laboratory, Department of Cell and Molecular Biology, Uppsala University, Husargatan 3, Box 596, SE-75124 Uppsala, Sweden

${ }^{\ddagger}$ Fjordforsk A.S., Institute for Science and Technology, Midtun, 6894 Vangsnes, Norway

${ }^{\S}$ Department of Biological Science and Engineering, School of Chemistry and Biological Engineering, University of Science and Technology Beijing, 100083 Beijing, China

"COSMOlogic GmbH \& Co. KG, Imbacher Weg 46, D-51379 Leverkusen, Germany

${ }^{\perp}$ Institute of Physical and Theoretical Chemistry, University of Regensburg, 93053 Regensburg, Germany

\section{Supporting Information}

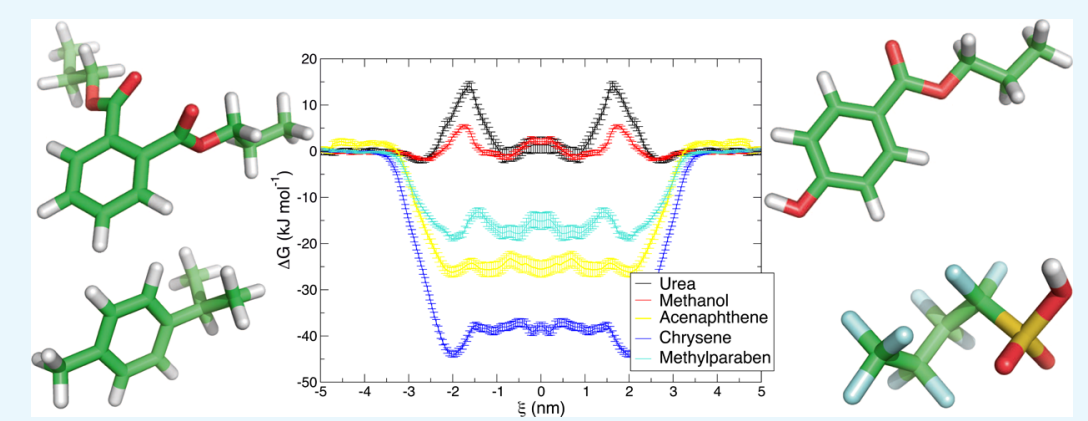

ABSTRACT: The partitioning of compounds between aqueous and other phases is important for predicting toxicity. Although thousands of octanol-water partition coefficients have been measured, these represent only a small fraction of the anthropogenic compounds present in the environment. The octanol phase is often taken to be a mimic of the inner parts of phospholipid membranes. However, the core of such membranes is typically more hydrophobic than octanol, and other partition coefficients with other compounds may give complementary information. Although a number of (cheap) empirical methods exist to compute octanol-water $\left(\log k_{\mathrm{OW}}\right)$ and hexadecane-water $\left(\log k_{\mathrm{HW}}\right)$ partition coefficients, it would be interesting to know whether physics-based models can predict these crucial values more accurately. Here, we have computed log $k_{\mathrm{OW}}$ and $\log k_{\mathrm{HW}}$ for 133 compounds from seven different pollutant categories as well as a control group using the solvation model based on electronic density (SMD) protocol based on Hartree-Fock (HF) or density functional theory (DFT) and the COSMO-RS method. For comparison, XlogP3 ( $\log k_{\mathrm{OW}}$ ) values were retrieved from the PubChem database, and KowWin log $k_{\mathrm{OW}}$ values were determined as well. For 24 of these compounds, $\log k_{\mathrm{OW}}$ was computed using potential of mean force (PMF) calculations based on classical molecular dynamics simulations. A comparison of the accuracy of the methods shows that COSMO-RS, KowWin, and XlogP3 all have a root-mean-square deviation (rmsd) from the experimental data of $\approx 0.4$ log units, whereas the SMD protocol has an rmsd of $1.0 \mathrm{log}$ units using HF and 0.9 using DFT. PMF calculations yield the poorest accuracy ( $\mathrm{rmsd}=1.1 \mathrm{log}$ units). Thirty-six out of 133 calculations are for compounds without known log $k_{\mathrm{OW}}$, and for these, we provide what we consider a robust prediction, in the sense that there are few outliers, by averaging over the methods. The results supplied may be instrumental when developing new methods in computational ecotoxicity. The log $k_{\mathrm{HW}}$ values are found to be strongly correlated to $\log k_{\mathrm{OW}}$ for most compounds.

\section{INTRODUCTION}

The chemical properties, biochemical interference, and biopersistence of environmental pollutants are critical factors for toxicology programs and strategies such as the REACH program and Tox $21 \mathrm{c} .{ }^{1-3}$ It is necessary to label, classify, and predict toxicological properties of chemicals so as to develop evidence-based environmental health and safety (EHS) standards for new and emerging compounds. ${ }^{4-6}$ As many new and emerging compounds and pollutants are known to pose serious environmental and health risks, ${ }^{7}$ effective and inexpensive modes of the assessment of chemical and toxicological properties are required. In this study, we use a number of computational chemistry methods to estimate the octanolwater $\left(\log k_{\mathrm{OW}}\right)$ and hexadecane-water $\left(\log k_{\mathrm{HW}}\right)$ partition

Received: May 3, 2019

Accepted: June 27, 2019

Published: August 12, 2019 
coefficients in order to get insight into membrane permeability. The modeling methods are applied to 133 compounds from the following categories: haloalkanes, haloaromatics, polycyclic aromatic hydrocarbons (PAHs), polycyclic biphenyls (PCBs), perfluorinated compounds (PFCs), parabens (PRBs), and phthalates (PHTs). All of these compounds belong to ubiquitous pollutant categories. ${ }^{7}$

The $\log k_{\mathrm{OW}}$ value approximates a compound's potential to partition into membranes, which is indirectly related to toxicity, because for most modes of action compounds have to cross a cell membrane. $\log k_{\mathrm{OW}}$ values therefore represent a cornerstone in pharmaceutical as well as environmental chemistry and toxicology, and it is important to determine $\log k_{\mathrm{OW}}$ for pollutants. Indeed, a toxicity profile including $\log k_{\mathrm{OW}}$ has to be determined before a chemical is allowed to enter the market in Europe, the United States of America, and Japan. ${ }^{8}$ However, for many emerging pollutants, the log $k_{\text {OW }}$ have not been resolved, ${ }^{9}$ and questions on their toxicity as well as regulatory decisions are still pending. ${ }^{10-13}$ In addition, there have been reports of disagreements in $\log k_{\mathrm{OW}}$ measurements. ${ }^{8}$ Indeed, experimental numbers for physicochemical observables may need more scrutiny in general. ${ }^{14} \mathrm{~A}$ number of recent experimental studies have focused on the compound classes studied here, for example, Quinn et al. studied partitioning of PCBs into different phases, ${ }^{15}$ while Xiang and co-workers focused on $\log k_{\mathrm{OW}}$ of perfluorated carboxylic acids, ${ }^{16}$ but for numerous compounds no reliable measurements are available. In general, the error in experimental measurements of $\log k_{\mathrm{OW}}$ varies from 0.1 to $1 \log$ units. $^{17}$

There is a large body of work related to measurement or prediction of $\log k_{\mathrm{OW}}$ based on, for instance, quantitative structure-property relationships (QSPRs). ${ }^{18,19}$ Although efforts into developing better experimental methods are ongoing, ${ }^{20}$ more research is focused on computational predictions ranging from coarse-grained simulations ${ }^{21}$ to analytical reference interaction site model theory, ${ }^{22}$ to quantum chemistry, ${ }^{23}$ to machine learning ${ }^{24,25}$ and other empirical methods. ${ }^{26,27}$ Interestingly, $\log k_{\mathrm{OW}}$ have been used to parameterize models for dissipative particle dynamics simulations as well. ${ }^{28}$ Simultaneously, there are still new efforts to measure new physicochemical data including $\log k_{\mathrm{OW}}$ with improvement of QSPR methods being one of the reasons. ${ }^{29}$ This is important as experimental databases are known to have errors in everything from names and structures to physicochemical properties. ${ }^{14,30,31}$

Environmental and toxicological sciences can, in principle, benefit from using the tools of computational chemistry to determine $\log k_{\mathrm{OW}}$ values and other properties of new chemicals to facilitate the differentiation of pollutants from innocuous chemicals. $^{23,24,32-39}$ In this study, we compare three computational approaches to calculate $\log k_{\mathrm{OW}}$ of 133 pollutant compounds, and, in addition, the $\log k_{\mathrm{HW}}$ are computed using two of the methods. The results are compared to experimental data as well as the XlogP prediction method ${ }^{40,41}$ and the widely used KowWin ( $k_{\mathrm{OW}}$-Windows), ${ }^{42,43}$ which is part of the EPI Suite. $^{44}$

\section{METHODS}

One hundred and thirty-three compounds were selected from seven pollutant categories, haloalkanes, haloaromatics, PAHs, polychlorinated biphenyls (PCBs), PFCs, PRBs, PHTs, and a control category. All compounds and computed values are listed in Table S1. Initial 3D coordinates of the pollutants were taken, if available, from the PubChem database directly, ${ }^{45}$ and if not available, the structures were built manually using Discovery Studio 4.5 Visualizer, followed by $\mathrm{AM}^{46}$ and $\mathrm{PM}^{47}$ optimizations in the gas phase using the Gaussian $09^{48}$ software. It should be noted that a varying number of the compounds studied in this paper have been used for parameterizing the methods used here. Experimental $\log k_{\mathrm{OW}}$ and $\log k_{\mathrm{HW}}$ values were taken from a number of sources (Table S1). In some cases, the partition coefficients were determined from the difference between solvation free energies in water and octanol, alternatively water and hexadecane. ${ }^{49}$ Some of the $\log k_{\mathrm{HW}}$ were taken from Hafkenscheid and Tomlinson who specify that the solvent is "aliphatic alkane" ${ }^{50}$ All results can be visualized on the (http://virtualchemistry.org) website. ${ }^{51}$ It should be noted that many papers in the literature refer to computed data as experimental or even present $\log k_{\mathrm{OW}}$ without any reference whatsoever.

2.1. SMD Calculations. Solvation model based on electronic density (SMD) calculations were performed with the Gaussian $09^{48}$ or the Gaussian $16^{52}$ software. Optimizations and frequency calculations were carried out for all the pollutants in the liquid phase with SMD solvent models (i.e., water, $n$ octanol, and $n$-hexadecane $)^{49}$ and in the gas phase separately at the HF $/ 6-31+G(d, p)^{53}$ level of theory. The LANL2DZdp-ECP basis set ${ }^{54}$ was used for iodine atoms, as this has shown to yield reasonable results in other studies. ${ }^{55}$ The abbreviation HartreeFock (HF) will be used for these calculations in the remainder of this work. In earlier work, ${ }^{56}$ a number of levels of theory were used, and it was found that both HF and density functional theory (DFT) are predicting numbers that are too low, which could be due to not only the basis set size applied but also the method. In order to distinguish these two possibilities, a further set of calculations was done using the BP86 functional ${ }^{57,58}$ (denoted as BP86 in what follows). The solvation free energy of pollutant molecules was defined to be the difference in the free energy of solute calculated in the liquid phase and in the gas phase. ${ }^{56,59,60}$ The partition coefficients were computed from the differences in solvation free energy of each compound in water, 1 -octanol, and $n$-hexadecane. This approximation ignores the fact that octanol is significantly hydrated, the solubility of water in octanol being $48.8 \mathrm{~g} / \mathrm{kg}$ at room temperature. ${ }^{61}$ The Minnesota solvation database ${ }^{62}$ has been used to develop and tune the SMD method, and some compounds from the database are used here.

2.2. COSMO-RS Calculations. COSMO-RS, that is, the conductor-like screening model for realistic solvation, ${ }^{63-65}$ is a quantum chemically based approach to predict thermodynamic equilibrium properties of molecules in liquids. It starts from polarization charge densities of solute and solvent molecules, which arise if the molecules are embedded in a virtual conductor. These can be efficiently calculated using DFT combined with the conductor-like screening model (COSMO) ${ }^{66}$ which is available in most quantum chemical programs. The TURBOMOLE program ${ }^{67}$ with a Becke-Perdew functional ${ }^{57,58}$ and a TZVPD basis set ${ }^{68}$ was used for these calculations, together with the default COSMO parameters in TURBOMOLE. On the basis of the individual COSMO results of solutes and solvent molecules, the COSMO-RS method expresses the specific interactions of molecules in a liquid system, that is, electrostatic interactions and hydrogen bonding, pairwise, local interactions of surface segments quantified by the COSMO polarization charge densities $\sigma$ of the interacting segments. By an efficient and accurate statistical thermodynamics calculation for the interacting surfaces, the chemical potentials and free energies of 
Table 1. Statistics for Prediction of $\log k_{\text {ow }}$ per Method and Compound Class and Number of (Neutral) Compounds Included Is Determined by the Availability of Experimental Data ${ }^{a}$

\begin{tabular}{|c|c|c|c|c|c|c|c|c|c|c|c|c|}
\hline \multirow[b]{2}{*}{ class } & \multicolumn{4}{|c|}{$\mathrm{HF}$} & \multicolumn{4}{|c|}{ BP86 } & \multicolumn{4}{|c|}{ COSMO-RS } \\
\hline & $N$ & $r^{2}$ & $\mathrm{rmsd}$ & MSE & $N$ & $r^{2}$ & $\mathrm{rmsd}$ & MSE & $N$ & $r^{2}$ & rmsd & MSE \\
\hline control & 12 & 0.98 & 0.57 & -0.33 & 12 & 0.99 & 0.43 & -0.08 & 12 & 0.99 & 0.22 & -0.04 \\
\hline haloalkane & 8 & 0.98 & 0.42 & 0.37 & 8 & 0.97 & 0.46 & 0.41 & 8 & 0.98 & 0.18 & 0.01 \\
\hline haloaromatic & 12 & 0.99 & 0.47 & -0.44 & 12 & 0.67 & 0.63 & -0.11 & 12 & 0.74 & 0.42 & -0.05 \\
\hline $\mathrm{PAH}$ & 17 & 0.89 & 1.51 & -1.35 & 17 & 0.71 & 1.36 & -1.15 & 17 & 0.93 & 0.41 & -0.18 \\
\hline РCB & 23 & 0.87 & 0.69 & -0.64 & 23 & 0.86 & 0.51 & -0.44 & 23 & 0.88 & 0.30 & -0.19 \\
\hline PFC & 6 & 0.88 & 0.38 & -0.13 & 6 & 0.54 & 0.60 & -0.09 & 6 & 0.67 & 0.89 & 0.76 \\
\hline PRB & 7 & 0.98 & 1.82 & -1.81 & 7 & 0.95 & 1.77 & -1.75 & 7 & 0.99 & 0.31 & -0.15 \\
\hline PHT & 12 & 0.83 & 1.09 & -0.42 & 12 & 0.93 & 1.00 & -0.76 & 12 & 0.99 & 0.56 & 0.47 \\
\hline \multirow[t]{2}{*}{ all } & 97 & 0.87 & 0.99 & -0.64 & 97 & 0.87 & 0.92 & -0.52 & 97 & 0.96 & 0.42 & 0.01 \\
\hline & \multicolumn{4}{|c|}{$\mathrm{X} \log \mathrm{P} 3$} & \multicolumn{4}{|c|}{ KowWin } & \multicolumn{4}{|c|}{ GAFF-ESP } \\
\hline class & $N$ & $r^{2}$ & $\mathrm{rmsd}$ & MSE & $N$ & $r^{2}$ & $\mathrm{rmsd}$ & MSE & $N$ & $r^{2}$ & $\mathrm{rmsd}$ & MSE \\
\hline control & 12 & 0.98 & 0.49 & -0.06 & 12 & 0.99 & 0.38 & -0.10 & 8 & 0.98 & 0.84 & 0.73 \\
\hline haloalkane & 8 & 0.98 & 0.17 & 0.00 & 8 & 0.96 & 0.23 & -0.00 & 3 & 1.00 & 1.41 & 1.23 \\
\hline haloaromatic & 12 & 1.00 & 0.07 & 0.02 & 12 & 0.88 & 0.29 & 0.01 & 2 & 1.00 & 1.29 & 1.27 \\
\hline $\mathrm{PAH}$ & 17 & 0.92 & 0.37 & 0.03 & 17 & 0.92 & 0.37 & -0.03 & 2 & 1.00 & 0.72 & 0.66 \\
\hline PCB & 23 & 0.89 & 0.38 & 0.18 & 23 & 0.84 & 0.60 & 0.31 & & & & \\
\hline PFC & 6 & 0.94 & 0.32 & 0.21 & 6 & 0.84 & 0.40 & -0.22 & 4 & 0.98 & 1.18 & 1.17 \\
\hline PRB & 7 & 1.00 & 0.03 & 0.01 & 7 & 0.99 & 0.11 & -0.01 & 5 & 0.89 & 1.23 & 0.87 \\
\hline PHT & 12 & 0.98 & 0.40 & 0.21 & 12 & 0.99 & 0.38 & 0.24 & & & & \\
\hline all & 97 & 0.98 & 0.34 & 0.09 & 97 & 0.97 & 0.41 & 0.07 & 24 & 0.93 & 1.10 & 0.93 \\
\hline
\end{tabular}

the molecules in pure and mixed solvents are calculated. For the current project, standard COSMO-RS calculations have been performed with the COSMOtherm program with the BP TZVPD FINE 18 parameterization. ${ }^{69}$ This means that the conformations and geometries used in the COSMO-RS calculations for the solutes and solvents were generated and handled as described in Klamt et al. ${ }^{70}$ For compounds, for which multiple conformations are relevant, in each solvent the free energy is calculated from the logarithm of the conformational partition function, leading to a multiconformational treatment which would be cumbersome in methods like SMD. log $k_{\mathrm{OW}}$ values of 16 of the compounds studied here were used in tuning the COSMO-RS code.

2.3. XlogP3 and KowWin. The $\mathrm{Xlog} P 3$ algorithm first searches for the most similar compound in the database, and if there is no full hit, the differences in the structures are accounted for by an incremental method. ${ }^{41}$ The values for the compounds studied here were downloaded from PubChem ${ }^{71}$ (Table S1). The algorithm yielded a root-mean-square deviation ( $\mathrm{rmsd}$ ) of $0.41 \mathrm{log}$ units for 8199 compounds in the original paper. ${ }^{41} \mathrm{~A}$ significant fraction of the compounds studied here is in the training set for XlogP3.

KowWin $\log k_{\text {OW }}$ were computed based on an empirical atom and fragment contribution method ${ }^{42}$ by the widely used EPI Suite. $^{43,44}$

2.4. Potential of Mean Force Calculations. Rectangular boxes containing 313 molecules of 1-octanol and 2627 water molecules were built where the 1-octanol fraction was slightly solvated (see analysis in Results and Discussion). This box was equilibrated for $2 \mathrm{~ns}$ in order to obtain a stable biphasic system. Pollutant input files were generated as described above. The generalized Amber force field $\left(\mathrm{GAFF}^{72}\right)$ was used for 1-octanol and all pollutant compounds. Charges for the pollutants were computed from the electrostatic potential using the MerzKollman procedure ${ }^{73,74}$ in Gaussian $16,{ }^{52}$ computed using DFT
$\left(\mathrm{B} 3 \mathrm{LYP}^{57,75-77}\right)$ combined with the aug-cc-pVTZ basis set. $^{78-80}$ The compounds are part of the Alexandria database, ${ }^{31}$ and Gaussian log files are available for download at Zenodo. ${ }^{81}$ The TIP3P water model ${ }^{82}$ was used. The GROMACS 2016 software package ${ }^{83,84}$ was used for all simulations. Long-range Coulomb interactions and Lennard-Jones ( $\mathrm{LJ}$ ) interactions were treated using the particle-mesh Ewald method (PME) ${ }^{85,86} \mathrm{LJ}$ PME was used because it has been shown that the omission of long-range LJ interactions leads to incorrect surface tensions of liquids ${ }^{87-89}$ and biological membranes ${ }^{86}$ and, in addition, has an effect on protein aggregation at high protein concentrations in simulations. ${ }^{90}$ Constraints were used on all chemical bonds to hydrogen atoms, applying the LINCS algorithm, ${ }^{91}$ allowing a 1 $\mathrm{fs}$ integration time step. Temperature coupling in production simulations was applied using the v-rescale algorithm ${ }^{92}$ with a time constant of $0.5 \mathrm{ps}$. The pressure was controlled using the Parrinello-Rahman algorithm ${ }^{93}$ with a time constant of $10 \mathrm{ps,}$ using the semi-isotropic scheme where the direction orthogonal to the interface is coupled separately from the other two directions. 2 ns simulations were performed "pulling" the pollutant through in the 1-octanol water box (see Movie M1) perpendicular to the water/1-octanol interface. We note that in the PMF method explicit water molecules do enter the octanol phase and contribute to the energy profile as discussed below. As the resulting numbers are in principle a property of the force field only, they will be denoted GAFF-ESP because electrostatic potential-derived charges were used.

\section{RESULTS AND DISCUSSION}

The prediction of $\log k_{\mathrm{OW}}$ values is summarized quantitatively in Table 1 for each of the classes of compounds. The lowest rmsd from experimental data for all compounds is obtained for XlogP3, COSMO-RS, and KowWin (all about 0.4 log unit), followed by the SMD method (HF: 1.0 and BP86: 0.9 log units) and the potential of mean force (PMF) calculations (1.1 log 
Table 2. Predictions for $\log k_{\mathrm{OW}}$ from Multiple Calculations for Neutral Compounds Where No Experimental Data Are Available ${ }^{a}$

\begin{tabular}{|c|c|c|c|c|c|c|c|}
\hline compound & category & HF & BP86 & COSMO-RS & $\mathrm{X} \log \mathrm{P} 3$ & KowWin & average \\
\hline$(2 R)$-1,2-dibromo-3-chloropropane & haloalkane & 3.03 & 3.17 & 2.30 & 2.40 & & $2.7(0.4)$ \\
\hline 1,2,3,4,5-pentafluoro-6-(2,3,4,5,6-pentafluorophenyl)benzene & haloaromatic & 3.73 & 4.36 & 5.84 & 4.60 & 5.76 & $4.9(0.9)$ \\
\hline 5-methylchrysene & $\mathrm{PAH}$ & 4.40 & 4.71 & 5.76 & 6.00 & 6.07 & $5.4(0.7)$ \\
\hline $7 H$-benzo $[c]$ fluorene & $\mathrm{PAH}$ & 4.17 & 4.39 & 5.15 & 5.70 & 5.19 & $4.9(0.6)$ \\
\hline benzo $[j]$ fluoranthene & $\mathrm{PAH}$ & 4.39 & 4.69 & 5.83 & 6.40 & 6.11 & $5.5(0.8)$ \\
\hline cyclopenta $[c d]$ pyrene & $\mathrm{PAH}$ & 4.03 & 4.31 & 5.12 & 5.50 & 5.70 & $4.9(0.7)$ \\
\hline indeno $[1,2,3-c d]$ pyrene & $\mathrm{PAH}$ & 4.72 & 5.12 & 6.22 & 7.00 & 6.70 & $6.0(0.9)$ \\
\hline 1,2,3,4,5-pentachloro-6-(2,3,4,6-tetrachlorophenyl)benzene & РCB & 7.45 & 7.76 & 7.72 & 8.20 & 9.56 & $8.1(0.8)$ \\
\hline 1,2,3,5-tetrachloro-4-(2,3,5,6-tetrachlorophenyl)benzene & PCB & 6.98 & 7.27 & 7.29 & 7.70 & 8.91 & $7.6(0.7)$ \\
\hline 2,2,3,3,4,4,4-heptafluorobutanoic acid & PFC & 1.18 & 0.98 & 2.88 & 2.20 & 2.14 & $1.9(0.8)$ \\
\hline (3R)-2,2,3-trifluoro-3-(trifluoromethyl)oxirane & PFC & 1.52 & 1.67 & 2.94 & 2.10 & 1.72 & $2.0(0.6)$ \\
\hline 2,3,4,5,6-pentafluorobenzoic acid & PFC & 1.99 & 1.42 & 2.82 & 2.00 & 1.78 & $2.0(0.5)$ \\
\hline$(4 R, 6 S)-1,1,2,2,3,3,4,5,5,6$-decafluoro-4,6-bis(trifluoromethyl)cyclohexane & PFC & 4.55 & 4.42 & 5.31 & 5.40 & 6.02 & $5.1(0.6)$ \\
\hline $\begin{array}{l}\text { 2,2,3,3,4,4,5,5,6,6,7,7,8,8,9,9,10,10,11,11,12,12,12-tricosafluorododecanoic } \\
\text { acid }\end{array}$ & PFC & 4.43 & 5.55 & 7.74 & 7.60 & 7.49 & $6.6(1.4)$ \\
\hline 2,2,3,3,4,4,5,5,6,6,7,7,7-tridecafluoroheptanoic acid & PFC & 2.54 & 2.78 & -1.82 & 4.30 & 4.15 & $2.4(2.3)$ \\
\hline 1,1,2,2,3,3,4,4,5,5,6,6,6-tridecafluorohexane-1-sulfonic acid & PFC & 0.79 & 1.21 & 5.12 & 3.70 & 3.16 & $2.8(1.7)$ \\
\hline 2,2,3,3,4,4,5,5,6,6,6-undecafluorohexanoic acid & PFC & 2.30 & 1.87 & 4.20 & 3.60 & 3.48 & $3.1(0.9)$ \\
\hline $1,1,1,2,2,3,3,4,5,5,5$-undecafluoro-4-(trifluoromethyl)pentane & PFC & 4.09 & 4.26 & 4.89 & 5.10 & 5.02 & $4.7(0.5)$ \\
\hline 2,2,3,3,4,4,5,5,6,6,7,7,8,8,9,9,9-heptadecafluorononanoic acid & PFC & 3.09 & 4.64 & 6.16 & 5.60 & 5.48 & $5.0(1.1)$ \\
\hline 2,2,3,3,4,4,5,5,5-nonafluoropentanoic acid & PFC & 1.01 & 1.80 & 3.50 & 2.90 & 2.81 & $2.4(0.9)$ \\
\hline $\begin{array}{l}\text { 1,1,2,2,3,3,4,4,5,5,5-undecafluoro- } N, N \text {-bis }(1,1,2,2,3,3,4,4,5,5,5 \text { - } \\
\text { undecafluoropentyl)pentan-1-amine }\end{array}$ & PFC & 8.21 & 8.39 & 10.83 & 11.90 & 10.21 & $9.9(1.5)$ \\
\hline $2,2,3,3,4,4,5,5,6,6,7,7,8,8,9,9,10,10,11,11,11$-henicosafluoroundecanoic acid & PFC & 3.63 & 4.04 & 7.44 & 6.90 & 6.82 & $5.8(1.6)$ \\
\hline 2-methylpropyl 4-hydroxybenzoate & PRB & 1.74 & 2.05 & 3.17 & 3.40 & 3.40 & $2.8(0.8)$ \\
\hline propan-2-yl 4-hydroxybenzoate & PRB & 1.07 & 1.58 & 2.73 & 2.80 & 2.91 & $2.2(0.8)$ \\
\hline octyl 4-hydroxybenzoate & PRB & 4.05 & 3.70 & 5.55 & 5.40 & 5.43 & $4.8(0.8)$ \\
\hline pentyl 4-hydroxybenzoate & $\mathrm{PRB}$ & 1.91 & 2.55 & 3.88 & 3.80 & 3.96 & $3.2(0.9)$ \\
\hline 1-O-butyl 2-O-cyclohexyl benzene-1,2-dicarboxylate & PHT & 4.36 & 4.64 & 5.30 & 5.30 & 5.41 & $5.0(0.5)$ \\
\hline dicyclohexyl benzene-1,2-dicarboxylate & PHT & 4.17 & 5.00 & 5.66 & 5.20 & 6.20 & $5.2(0.7)$ \\
\hline bis(7-methyloctyl) benzene-1,2-dicarboxylate & PHT & 7.75 & 8.15 & 9.80 & 9.60 & 9.37 & $8.9(0.9)$ \\
\hline diundecyl benzene-1,2-dicarboxylate & PHT & 9.34 & 11.85 & 12.33 & 12.30 & 11.49 & $\begin{array}{l}11.5 \\
(1.2)\end{array}$ \\
\hline butyl decyl phthalate & PHT & 5.68 & 6.61 & 8.05 & 8.00 & 7.56 & $7.2(1.0)$ \\
\hline bis(5-methylhexyl) benzene-1,2-dicarboxylate & PHT & 5.58 & 6.56 & 7.69 & 7.40 & 7.41 & $6.9(0.8)$ \\
\hline bis(4-methylpentyl) benzene-1,2-dicarboxylate & PHT & 4.96 & 5.61 & 6.53 & 6.30 & 6.43 & $6.0(0.6)$ \\
\hline bis(11-methyldodecyl) benzene-1,2-dicarboxylate & PHT & 11.77 & 12.61 & & 13.90 & 13.30 & $\begin{array}{r}12.9 \\
(0.8)\end{array}$ \\
\hline bis(9-methyldecyl) benzene-1,2-dicarboxylate & PHT & 8.23 & 9.18 & 11.99 & 11.70 & & $\begin{array}{l}10.3 \\
(1.7)\end{array}$ \\
\hline bis(2-propylheptyl) benzene-1,2-dicarboxylate & PHT & 8.12 & 9.05 & 10.72 & 9.60 & 10.36 & $9.6(1.0)$ \\
\hline
\end{tabular}

unit). Both SMD methods systematically underestimate $\log k_{\mathrm{OW}}$ with a mean signed error (MSE) of $\approx-0.6$. The HF method is known to overpolarize compounds; ${ }^{31,94}$ however, this should not be the case for BP86, and therefore, there may be other contributing factors. The GAFF-ESP calculations on the other hand overestimate $\log k_{\text {OW }}$ possibly due to lack of explicit polarizability. The results are skewed slightly by outliers in some of the compound classes, which will be discussed in some detail below. The XlogP3 rmsd is low due to the fact that some of compounds may be part of the database used for optimizing the algorithm. It should also be noted that the experimental error varies between 0.1 and $1.0 \mathrm{log}$ unit, with larger compound having larger uncertainty. ${ }^{17}$

The calculation times vary between less than a second for the QSPR methods to minutes for COSMO-RS to days for the DFT and HF methods to half a year for each of the PMFs. Although long calculation times may preclude high-throughput usage of the methods, it is important to establish the relative accuracies of the methods.

3.1. Control Compounds. The control class consists of a number of small polar and apolar compounds including aromatic compounds. They were chosen to have a range of $\log k_{\text {OW }}$ values, including negative ones and known experimental values. All methods except GAFF-ESP perform relatively well for this category with small MSE (Table 1).

3.2. Haloalkanes. The haloalkane group comprised a set of eight compounds (Table S1), for which $\log k_{\mathrm{OW}}$ predictions all yield high correlation coefficients and low rmsd (Table 1). Interestingly, SMD yields an almost perfect correlation coefficient $r^{2}$ of 0.98 ; however, all $\log k_{\mathrm{OW}}$ are overestimated by $0.42 \log$ units, while, in contrast, all other groups are underestimated systematically (Table 1 ). This result suggests that there is room for improvement with the parameterization of the 1-octanol solvent in the SMD model. The compounds chloromethane, chloroethane, and pentachloroethane were also 
evaluated using GAFF-ESP, and these are overestimated in all cases, in particular for 1,1,1,2,2-pentachloroethane. The reason for this discrepancy is likely associated with the force-field parameters, which are not specifically optimized for compounds such as haloalkanes. Addition of a virtual site to model halogen bonding, as present in other general force fields, ${ }^{95}$ might help resolve these issues to some extent.

3.3. Haloaromatic Compounds. $\log k_{\mathrm{OW}}$ for 12 haloaromatic compounds were predicted using the SMD and COSMO-RS methods, and for three of these, 1-chloro-3phenylbenzene and hexachlorobenzene predictions were done using GAFF-ESP as well. The results (Tables 1 and S1) show an rmsd between 0.4 and 0.5 for all methods. The rmsd for SMDBP86 is slightly higher than the other methods because of one compound, namely, 1-chloro-3-phenylbenzene (Table S1).

3.4. Polycyclic Aromatic Hydrocarbons. It should be relatively easy to predict $\log k_{\mathrm{OW}}$ for PAHs given their planar structures, lack of substituents, and their limited number of geometrical conformations. Indeed, our calculations display a reasonable agreement with empirical data (Table 1) with $r^{2}>$ 0.85 in all cases except SMD-BP86. However, both SMD methods have a few severe outliers $(-2 \log$ units, Table S1). It may be that larger PAHs $\left(>\mathrm{C}_{16}\right)$ which pertain higher $\log k_{\mathrm{OW}}$ values are more difficult to be predicted correctly using SMD as a result of their aromatic moment across the large planar structures. $^{32}$ 1,2-Dihydroacenaphthylene and chrysene were predicted using GAFF-ESP, yielding moderate overestimations in both cases (Table S1), in line with the overall trend (Table 1).

3.5. Polychlorinated Biphenyls. PCBs are predicted quite accurately by all methods, in particular COSMO-RS. In the case of SMD-HF, there is a MSE of $-0.64 \log$ units, -0.44 for SMDBP86. Given that PCBs have been present in the environment for a long time, ${ }^{7}$ there is a large amount of data available and only two predictions are made here (Table 2).

3.6. Perfluorinated Compounds. PFCs have an antipromiscuous chemistry, which makes these compounds associate with neither water nor octanol. This might lead to problems in experimental assessments too. Our predictions for the few compounds are quite close to the experimental values; however, the values in Table 2 vary a lot between the methods used here, with large standard deviation for a number of compounds. Hidalgo and colleagues ${ }^{96}$ recently reported $\log k_{\mathrm{OW}}$ values computed using SMD for medium-weight (up to 11 carbon atoms) linear PFCs and compare the results to empirical $\log k_{\text {OW }}$ from the KowWin program. ${ }^{42-44}$ They question some of the experimental data and also find systematic differences between the results obtained using purely empirical methods and the quantum chemistry-based SMD results. GAFF-ESP simulations were done for four PFCs from this set, namely, 1,1,1,2,2,2-hexafluoroethane, 1,1,1,2,2,3,3,3-octafluoropropane, 1,1,1,3,3,3-hexafluoropropan-2-ol, and 2,2,2-trifluoroacetic acid yielding overestimations of 1.2, 1.0, 1.4, and $1.1 \mathrm{log}$ units from the experiment, respectively. The PMF method with the used GAFF-ESP force field therefore does not improve on the accuracy of the SMD methods or COSMO-RS.

3.7. Parabens. The $\log k_{\mathrm{OW}}$ for $\mathrm{PRBs}$ are predicted accurately using COSMO-RS and for XlogP3 and KowWin as well. For SMD, a large systematic deviation (MSE of -1.8 log units) was found using both HF and BP86 methods. To our knowledge however, only one study has reported multiple theoretically predicted $\log k_{\text {Ow }}$ values for PRBs. ${ }^{97}$ In that work by Casoni and Sârbu, the most accurate method for calculation was found to be ACLogP, where methylparaben, ethylparaben, propylparaben, and butylparaben were predicted with a deviation of $0-0.22 \log$ units compared with experimental results from a study by Kitagawa and $\mathrm{Li}^{98}$ From the study by Casoni and Sârbu, it can be concluded that the larger the PRB molecule becomes, the higher the deviation from the empirical results. The results for SMD based on either HF or BP86 point more to a constant offset, however. Five of the PRBs were also studied using PMFs, namely, methyl 4-hydroxybenzoate, ethyl 4-hydroxybenzoate, propyl 4-hydroxybenzoate, butyl 4-hydroxybenzoate, and heptyl-4-hydroxybenzoate, which gave a lower rmsd than SMD (1.2 log units) but opposite sign of the MSE.

3.8. Phthalates. PHTs turned out to be difficult to predict with an rmsd $>0.9$ for both SMD methods (Table 1). For COSMO-RS, this category displays the largest (positive) MSE of all. The PHTs bear a ring moiety with two carbon chains attached and have therefore a large number of degrees of freedom, which may contribute entropically to the free energies of solvation and hence to the high rmsd. Although the PHT category contains many relatively large compounds, there is no clear correlation between molecular weight and error in the prediction.

3.9. log $k_{\text {ow }}$ Predictions. Table 2 displays computed log $k_{\mathrm{OW}}$ for 36 compounds. The averages over the five numbers are proposed to be the predicted values because the rmsd for the average for the compounds where there are experimental data is slightly lower than any of the methods by itself. Inoue and coworkers reported predictions of log $k_{\mathrm{OW}}$ for some large PFCs ${ }^{100}$ using the KowWin program. ${ }^{42-44}$ Their numbers are quite a bit higher than what is found here using SMD, but it seems that the $\log k_{\mathrm{OW}}$ of this category of compounds as well as PRBs and PHTs are underestimated systematically in SMD (Figure 1). For PAHs, the size-dependent underestimation is present for the SMD methods. Nevertheless, there is a good correspondence between the methods, and because of the physical approach used in SMD and COSMO-RS, it seems reasonable to assume

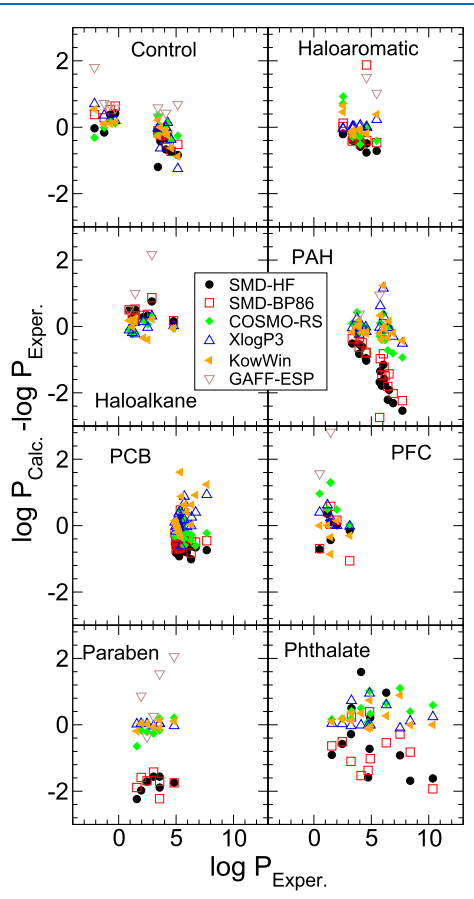

Figure 1. Correlation between experimental and calculated $\log P$ (residual) for six methods separately for all the classes considered. 
that the average numbers provided in Table 2 are good approximations.

The PMF calculations described here allow water to enter the octanol phase and in this manner influence the log $k_{\mathrm{OW}}$ through preferential solvation or, in principle, by binding alcohol groups in the octanol phase. An analysis of the amount of water in the octanol phase yields no difference depending on the solute: in all systems, approximately $6 \pm 1$ water molecule is found in the octanol phase.

3.10. $\log k_{\mathrm{HW}} \cdot \log k_{\mathrm{OW}}$ partition coefficients have been used in environmental analysis and for toxicity prediction for several decades, and various methods for calculating and determining $\log k_{\mathrm{OW}}$ empirically have been devised. However, some studies ${ }^{101,102}$ suggest that relying solely on the solubility of a compound in octanol and water may not yield a complete picture of the potential toxicity of the compound. For this reason, we have also performed predictions of the hexadecane $\left(\mathrm{C}_{16} \mathrm{H}_{34}\right)$ water partition coefficient $\log k_{\mathrm{HW}}\left(\right.$ Table S1). $\mathrm{C}_{16} \mathrm{H}_{34}$ has a higher capacity to solvate heavy apolar compounds, such as large PAHs and hydrocarbons and potentially also PFCs. ${ }^{50}$

All computed $\log k_{\mathrm{HW}}$ are given in Table $\mathrm{S} 1$ alongside a small number (12) of experimental data points obtained from the Minnesota solvation database ${ }^{62}$ (based on experimental data from, e.g., Abraham ${ }^{103}$ ) and from partition coefficients for water/aliphatic alkanes. ${ }^{50}$ Compared to these 12 data points, the predictions are within $1 \log$ unit for all compounds except for urea. Figure 2 shows the correlation between $\log k_{\mathrm{HW}}$ computed

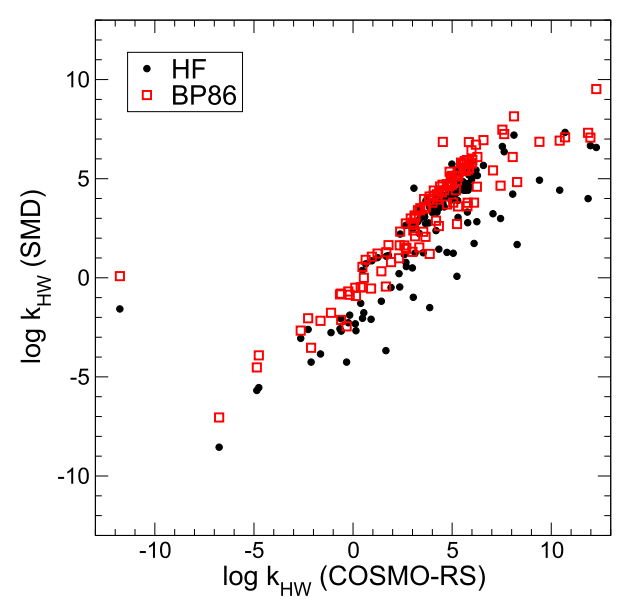

Figure 2. Comparison between $\log k_{\mathrm{HW}}$ computed using the COSMO$\mathrm{RS}$ ( $X$-axis) and the quantum chemical method SMD ( $Y$-axis) for the HF and BP86 methods.

using COSMO-RS and both SMD methods. When neglecting one outlier, 2,2,3,3,4,4,5,5,6,6,7,7,7-tridecafluoroheptanoic acid, the correlations $r^{2}=0.72$ for HF and 0.76 for BP86, respectively, with rmsd between the two methods of 2.4 (HF) and 1.1 (BP86) $\log$ units. Because both COSMO-RS and SMD contain empirical elements, it is difficult to pinpoint what could be the underlying reason for the discrepancies, but BP86 is much closer to COSMO-RS than HF. It may be, obviously, that less effort has gone into fine-tuning the parameterization of implicit solvent models for hexadecane than for water and octanol. Nevertheless, COSMO-RS is known to perform well for a range of solvents, ${ }^{104,105}$ while SMD also has been shown to outperform implicit solvent models based on empirical force fields. ${ }^{56}$ Another issue could be that the basis set is not sufficiently large for PFCs, but evaluation of basis sets is beyond the scope of this paper.

Figure 3 shows that the correlation plots for the PFCs, PRBs, and PHTs all have a slope close to one but an offset of -3 to 4 log units. For haloalkanes, haloaromatics, and PCBs, the difference between $\log k_{\mathrm{OW}}$ and $\log k_{\mathrm{HW}}$ is small as it is for the hydrophobic $\left(\log k_{\mathrm{OW}}>0\right)$ control compounds. The truly hydrophilic control compounds are much more soluble in octanol than in hexadecane. For most PAH compounds $\log k_{\mathrm{HW}}$ is slightly larger than $\log k_{\mathrm{OW}}$. These findings are in line with the wellknown result that more aliphatic compounds solvate more readily in lipid bilayers. ${ }^{106}$ This suggests that there is not a lot of extra information to be had from log $k_{\mathrm{HW}}$ calculations (or measurements) if the $\log k_{\mathrm{OW}}$ is known already.

\section{CONCLUSIONS}

Comparisons of empirical methods for computing $\log k_{\mathrm{OW}}$ have been published previously, including molecular modeling studies ${ }^{107,108}$ and more approximative models. ${ }^{109-113}$ Bannan et al. used separate free energy of solvation calculations in different solvents to obtain the $\log k_{\mathrm{OW}}{ }^{108}$ These authors obtained an rmsd of $\approx 1.6$ log units, slightly larger than the value found here (1.1, Table 1). Although the number of compounds is too small to draw any conclusion and the compounds studied are different, it might be worthwhile to study whether the biphasic system used here improves the predictive power. Benfenati and co-workers compared KowWin to a number of other software packages and found this package to be one of the most accurate ones. ${ }^{109}$ More recently, dos Reis and co-workers compared different prediction algorithms in a statistical analysis and found KowWin to be one of the most accurate ones. ${ }^{111}$ In contrast, Geisler et al., in a comparison of $\log k_{\mathrm{OW}}$ prediction for small compounds, found KowWin to be quite a bit less accurate than COSMOtherm, ${ }^{112}$ which is more in line with the results presented here. It is of interest that development of QSPR methods is ongoing, ${ }^{114}$ in part fueled by the finding that databases used to derive older QSPR methods from needed to be curated. ${ }^{30}$

In this study, we have derived $\log k_{\mathrm{OW}}$ values for a large set of compounds from different chemical classes using four computational methods. The quantum chemical SMD approach and the COSMO-RS methods were used to compute log $k_{\mathrm{OW}}$ and $\log$ $k_{\mathrm{HW}}$ for 133 compounds, while XlogP3 $\left(\log k_{\mathrm{OW}}\right)$ values were downloaded for reference and KowWin values computed using the EPI suite. By taking the average over four to five values, we provide what we consider accurate $\log k_{\text {Ow }}$ predictions for 36 compounds for which no experimental data are available (Table 2 ). Because the number of available experimental data points remains limited despite decades of measurements, we hope these numbers may be of use in environmental toxicity applications. Of the three methods used for the predictions, the SMD method systematically underestimates $\log k_{\text {OW }}$, while COSMO-RS and XlogP3 overestimate log $k_{\mathrm{OW}}$ slightly. COSMO-RS yields the most accurate predictions in the tests provided here.

For a number of difficult cases, molecular dynamics simulations were used to compute the PMF for transport through the octanol-water phase. The method has an rmsd from the experimental data of $\approx 1.1 \log$ units. However, if the large MSE ( $\approx 1.0$ log units) is subtracted from the results, the rmsd reduces to $0.8 \log$ units. The finding that the PMF method systematically overestimates $\log k_{\mathrm{OW}}$ could be related to a deficiency in either of the solvent models, although the TIP3P 


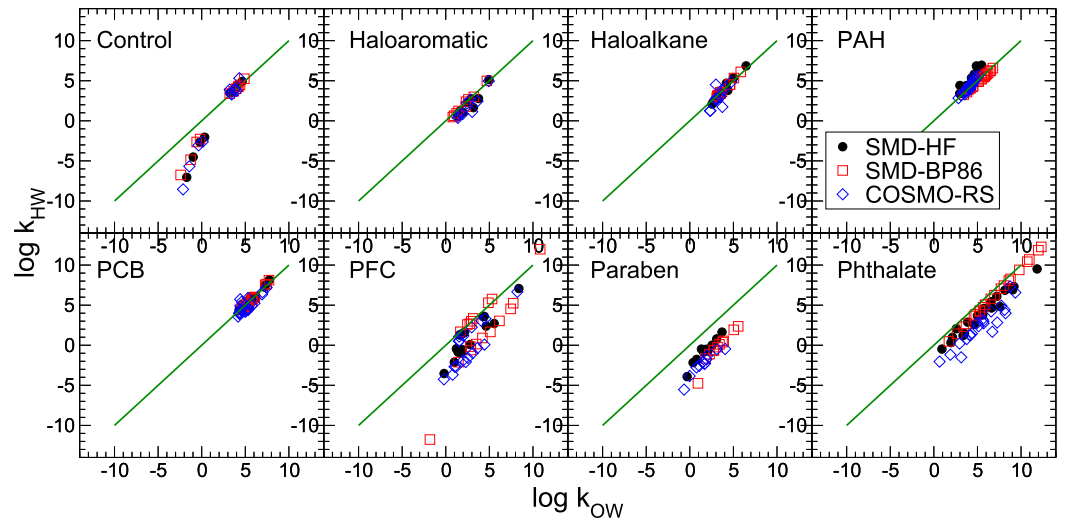

Figure 3. Comparison between $\log k_{\mathrm{OW}}$ and $\log k_{\mathrm{HW}}$ computed using the quantum chemical method SMD-HF and SMD-BP86 as well as COSMO-RS for all compounds. The green lines correspond to $\log k_{\mathrm{OW}}=\log k_{\mathrm{HW}}$, and it is plotted to guide the eye.

water model is known to reproduce solvation relatively well. ${ }^{104,105,115,116}$ Considering the computational cost, PMFs are not competitive because the quality of the predictions is not better than the cheaper methods. Of the other methods, SMD is relatively expensive with CPU requirements varying from minutes to days because of nonlinear scaling of quantum chemical calculations with system size. DFT is more CPU-time efficient than HF. COSMO-RS is quite a bit more efficient than SMD, while KowWin is virtually instantaneous. Nevertheless, with the present quality of predictions, it may be wise to apply more than one method. It should also be added that our results should not be extrapolated to compounds with chemical moieties far outside the range of compounds here.

\section{ASSOCIATED CONTENT}

\section{S Supporting Information}

The Supporting Information is available free of charge on the ACS Publications website at DOI: 10.1021/acsomega.9b01277.

IUPAC name, PubChem ID, CAS, category, log $k_{\mathrm{OW}}$ and $\log k_{\mathrm{HW}}$, free energies of solvation from for the compounds in the three different solvents computed using the SMD method (ZIP)

All computed and experimental $\log k_{\mathrm{OW}}$ and $\log k_{\mathrm{HW}}$ for the compounds (PDF)

Example simulation showing "pulling" the pollutant through in the 1-octanol water box perpendicular to the water/1-octanol interface (MOV)

\section{AUTHOR INFORMATION}

\section{Corresponding Author}

*E-mail: david.vanderspoel@icm.uu.se. Phone: +46 18 4714205 .

ORCID $\odot$

David van der Spoel: 0000-0002-7659-8526

Sergio Manzetti: 0000-0003-4240-513X

Haiyang Zhang: 0000-0002-2410-7078

Andreas Klamt: 0000-0002-5320-6219

\section{Funding}

The Swedish Research Council is acknowledged for a grant of computer time (SNIC2017-12-41) through the High-Performance Computing Center North in Umeå, Sweden.

Notes

The authors declare the following competing financial interest(s): Dr. Andreas Klamt declares to be the inventor of the COSMO-RS method and to develop and distribute COSMO-RS in the form of the COSMOtherm software commercially via his company, COSMOlogic.

\section{ABBREVIATIONS}

GROMACS, Groningen machine for chemical simulation; MD, molecular dynamics; PMF, potential of mean force; PHT, phthalates; PFC, perfluorinated compounds; PRB, parabens; $\mathrm{PAH}$, polycyclic aromatic hydrocarbon; HAA, haloalkanes; HAM, haloaromatics

\section{REFERENCES}

(1) Penman, M.; Banton, M.; Erler, S.; Moore, N.; Semmler, K. Olefins and chemical regulation in Europe: REACH. Chem.-Biol. Interact. 2015, 241, 59-65.

(2) Rovida, C.; Asakura, S.; Daneshian, M.; Hofman-Huether, H.; Leist, M.; Meunier, L.; Reif, D.; Rossi, A.; Schmutz, M.; Valentin, J.-P. Toxicity testing in the 21 st century beyond environmental chemicals. ALTEX 2015, 32, 171-181.

(3) Versonnen, B.; Tarazona, J. V.; Cesnaitis, R.; Sobanska, M. A.; Sobanski, T.; Bonnomet, V.; De Coen, W. Analysis of the ecotoxicity data submitted within the framework of the REACH Regulation. Sci. Total Environ. 2014, 475, 123-131.

(4) Helland, A.; Kastenholz, H.; Siegrist, M. Precaution in practice. J. Ind. Ecol. 2008, 12, 449-458.

(5) Schwarzman, M.; Wilson, M. Reshaping Chemicals Policy on Two Sides of the Atlantic: Ecosystem Impacts of Current Approaches and the Promise of Improved Sustainability through International Collaboration, 2010.

(6) Wombacher, W. There's Cologne in the Water: The Inadequacy of US Environmental Statutes to Address Emerging Environmental Contaminants. Colo. J. Int. Environ. Law Pol. 2010, $21,521$.

(7) Manzetti, S.; van der Spoel, E. R.; van der Spoel, D. Chemical Properties, Environmental Fate, and Degradation of Seven Classes of Pollutants. Chem. Res. Toxicol. 2014, 27, 713-737.

(8) Renner, R. The Kow Controversy. Environ. Sci. Technol. 2002, 36, $410 \mathrm{~A}-413 \mathrm{~A}$

(9) Caixach, J.; Bartolomé, A. Experiences from Surface Water Quality Monitoring; Springer, 2015; pp 269-281.

(10) Kirchhof, M. G.; de Gannes, G. C. The health controversies of parabens. Skin. Ther. Lett. 2013, 18, 5-7.

(11) Purcaro, G.; Moret, S.; Conte, L. S. Overview on polycyclic aromatic hydrocarbons: occurrence, legislation and innovative determination in foods. Talanta 2013, 105, 292-305.

(12) Surma, M.; Zieliński, H. What do we know about the risk arising from perfluorinated compounds. Pol. J. Environ. Stud. 2015, 24, 449457.

(13) Zota, A. R.; Calafat, A. M.; Woodruff, T. J. Temporal trends in phthalate exposures: findings from the National Health and Nutrition 
Examination Survey, 2001-2010. Environ. Health Perspect. 2014, 122, 235-241.

(14) Ghahremanpour, M. M.; van Maaren, P. J.; Ditz, J. C.; Lindh, R.; van der Spoel, D. Large-Scale Calculations of Gas Phase Thermochemistry: Enthalpy of Formation, Standard Entropy and Heat Capacity. J. Chem. Phys. 2016, 145, 114305.

(15) Quinn, C. L.; van der Heijden, S. A.; Wania, F.; Jonker, M. T. O. Partitioning of Polychlorinated Biphenyls into Human Cells and Adipose Tissues: Evaluation of Octanol, Triolein, and Liposomes as Surrogates. Environ. Sci. Technol. 2014, 48, 5920-5928.

(16) Xiang, Q.; Shan, G.; Wu, W.; Jin, H.; Zhu, L. Measuring log K-ow coefficients of neutral species of perfluoroalkyl carboxylic acids using reversed-phase high-performance liquid chromatography. Environ. Pollut. 2018, 242, 1283-1290.

(17) Sangster, J. Octanol-Water Partition Coefficients of Simple Organic Compounds. J. Phys. Chem. Ref. Data 1989, 18, 1111-1229.

(18) Katritzky, A. R.; Kuanar, M.; Slavov, S.; Hall, C. D.; Karelson, M.; Kahn, I.; Dobchev, D. A. Quantitative Correlation of Physical and Chemical Properties with Chemical Structure: Utility for Prediction. Chem. Rev. 2010, 110, 5714-5789.

(19) Lipinski, C. A.; Lombardo, F.; Dominy, B. W.; Feeney, P. J. Experimental and computational approaches to estimate solubility and permeability in drug discovery and development settings. Adv. Drug Delivery Rev. 2012, 64, 4-17.

(20) Low, Y. W.; Blasco, F.; Vachaspati, P. Optimised method to estimate octanol water distribution coefficient $(\log \mathrm{D})$ in a high throughput format. Eur. J. Pharm. Sci. 2016, 92, 110-116.

(21) Genheden, S. Predicting Partition Coefficients with a Simple AllAtom/Coarse-Grained Hybrid Model. J. Chem. Theory Comput. 2016, 12, 297-304.

(22) Huang, W.; Blinov, N.; Kovalenko, A. Octanol-Water Partition Coefficient from 3D-RISM-KH Molecular Theory of Solvation with Partial Molar Volume Correction. J. Phys. Chem. B 2015, 119, 55885597.

(23) Michalík, M.; Poliak, P.; Klein, E.; Lukeš, V. On the toxicity of para-substituted phenols and their quinone metabolites: Quantum chemical study. Chem. Phys. Lett. 2018, 709, 71-76.

(24) Riniker, S. Molecular Dynamics Fingerprints (MDFP): Machine Learning from MD Data To Predict Free-Energy Differences. J. Chem. Inf. Model. 2017, 57, 726-741.

(25) Safder, U.; Nam, K.; Kim, D.; Shahlaei, M.; Yoo, C. Quantitative structure-property relationship (QSPR) models for predicting the physicochemical properties of polychlorinated biphenyls (PCBs) using deep belief network. Ecotoxicol. Environ. Saf. 2018, 162, 17-28.

(26) Kim, T.; Park, H. Computational prediction of octanol-water partition coefficient based on the extended solvent-contact model. J. Mol. Graphics 2015, 60, 108-117.

(27) Toropov, A. A.; Toropova, A. P.; Cappelli, C. I.; Benfenati, E. CORAL: Model for octanol/water partition coefficient. Fluid Phase Equilib. 2015, 397, 44-49.

(28) Anderson, R. L.; Bray, D. J.; Ferrante, A. S.; Noro, M. G.; Stott, I. P.; Warren, P. B. Dissipative particle dynamics: Systematic parametrization using water-octanol partition coefficients. J. Chem. Phys. 2017, 147, 094503.

(29) Nicolas, C. I.; Mansouri, K.; Phillips, K. A.; Grulke, C. M.; Richard, A. M.; Williams, A. J.; Rabinowitz, J.; Isaacs, K. K.; Yau, A.; Wambaugh, J. F. Rapid experimental measurements of physicochemical properties to inform models and testing. Sci. Total Environ. 2018, 636, 901-909.

(30) Mansouri, K.; Grulke, C. M.; Richard, A. M.; Judson, R. S.; Williams, A. J. An automated curation procedure for addressing chemical errors and inconsistencies in public datasets used in QSAR modelling. SAR QSAR Environ. Res. 2016, 27, 911-937.

(31) Ghahremanpour, M. M.; van Maaren, P. J.; van der Spoel, D. The Alexandria Library: A Quantum Chemical Database of Molecular Properties for Force Field Development. Sci. Data 2018, 5, 180062.

(32) Manzetti, S. Handbook of Polycyclic Aromatic Hydrocarbons: Chemistry, Occurrence and Health Issues; Nova Sciences Publishers, 2012.
(33) Manzetti, S. Ecotoxicity of polycyclic aromatic hydrocarbons, aromatic amines, and nitroarenes through molecular properties. Environ. Chem. Lett. 2012, 10, 349-361.

(34) Deng, Z.; Lin, Z.; Zou, X.; Yao, Z.; Tian, D.; Wang, D.; Yin, D. Model of Hormesis and Its Toxicity Mechanism Based on Quorum Sensing: A Case Study on the Toxicity of Sulfonamides to Photobacterium phosphoreum. Environ. Sci. Technol. 2012, 46, $7746-7754$.

(35) Qu, R.; Liu, H.; Feng, M.; Yang, X.; Wang, Z. Investigation on Intramolecular Hydrogen Bond and Some Thermodynamic Properties of Polyhydroxylated Anthraquinones. J. Chem. Eng. Data 2012, 57, $2442-2455$.

(36) Manzetti, S.; Andersen, O. A molecular dynamics study of nanoparticle-formation from bioethanol-gasoline blend emissions. Fuel 2016, 183, 55-63.

(37) Sergio, M.; Behzadi, H.; Andersen, O.; van der Spoel, D. Fullerenes toxicity and electronic properties. Environ. Chem. Lett. 2013, 11, 105-118.

(38) Yamin, P.; Isele-Holder, R.; Leonhard, K. Predicting Octanol/ Water Partition Coefficients of Alcohol Ethoxylate Surfactants Using a Combination of Molecular Dynamics and the Conductor-like Screening Model for Realistic Solvents. Ind. Eng. Chem. Res. 2016, 55, 47824789.

(39) Yildirim, A.; Zhang, J.; Manzetti, S.; van der Spoel, D. Binding of Pollutants to Biomolecules: A Simulation Study. Chem. Res. Toxicol. 2016, 29, 1679-1688.

(40) Wang, R.; Fu, Y.; Lai, L. A new atom-additive method for calculating partition coefficients. J. Chem. Inf. Comput. Sci. 1997, 37, $615-621$.

(41) Cheng, T.; Zhao, Y.; Li, X.; Lin, F.; Xu, Y.; Zhang, X.; Li, Y.; Wang, R.; Lai, L. Computation of octanol- water partition coefficients by guiding an additive model with knowledge. J. Chem. Inf. Model. 2007, $47,2140-2148$.

(42) Meylan, W. M.; Howard, P. H. Estimating log P with atom/ fragments and water solubility with log P. Perspect. Drug Discovery Des. 2000, 19, 67-84.

(43) Estimation Programs Interface Suite for Microsoft Windows, v 4.11; United States Environmental Protection Agency: Washington, DC, U.S.A., 2018.

(44) Card, M. L.; Gomez-Alvarez, V.; Lee, W.-H.; Lynch, D. G.; Orentas, N. S.; Lee, M. T.; Wong, E. M.; Boethling, R. S. History of EPI Suite and future perspectives on chemical property estimation in US Toxic Substances Control Act new chemical risk assessments. Environ. Sci.: Processes Impacts 2017, 19, 203-212.

(45) Kim, S.; Thiessen, P. A.; Bolton, E. E.; Chen, J.; Fu, G.; Gindulyte, A.; Han, L.; He, J.; He, S.; Shoemaker, B. A.; Wang, J.; Yu, B.; Zhang, J.; Bryant, S. H. PubChem Substance and Compound databases. Nucleic Acids Res. 2016, 44, D1202-D1213.

(46) Dewar, M. J. S.; Zoebisch, E. G.; Healy, E. F.; Stewart, J. J. P. Development and use of quantum mechanical molecular models. 76 . AM1: a new general purpose quantum mechanical molecular model. J. Am. Chem. Soc. 1985, 107, 3902-3909.

(47) Stewart, J. J. P. Optimization of parameters for semiempirical methods V: Modification of NDDO approximations and application to 70 elements. J. Mol. Model. 2007, 13, 1173-1213.

(48) Frisch, M. J.; et al. Gaussian 09, Revision B.01; Gaussian Inc: Wallingford, CT, 2009.

(49) Marenich, A. V.; Cramer, C. J.; Truhlar, D. G. Universal Solvation Model Based on Solute Electron Density and on a Continuum Model of the Solvent Defined by the Bulk Dielectric Constant and Atomic Surface Tensions. J. Phys. Chem. B 2009, 113, 6378-6396.

(50) Hafkenscheid, T. L.; Tomlinson, E. Correlations between alkane/water and octan-1-ol/ water distribution coefficients and isocratic reversed-phase liquid chromatographic capacity factors of acids, bases and neutrals. Int. J. Pharm. 1983, 16, 225-239.

(51) van der Spoel, D.; van Maaren, P. J.; Caleman, C. GROMACS Molecule \& Liquid Database. Bioinformatics 2012, 28, 752-753.

(52) Frisch, M. J.; et al. Gaussian 16, Revision A.03; Gaussian Inc: Wallingford CT, 2016. 
(53) Hehre, W. J.; Radom, L.; Schleyer, P. v. R.; Pople, J. A. Ab Initio Molecular Orbital Theory; Wiley-Interscience: New York, 1986.

(54) Check, C. E.; Faust, T. O.; Bailey, J. M.; Wright, B. J.; Gilbert, T. M.; Sunderlin, L. S. Addition of Polarization and Diffuse Functions to the Lanl2dz Basis Set for P-Block Elements. J. Phys. Chem. A 2001, 105, $8111-8116$

(55) Beale, T. M.; Chudzinski, M. G.; Sarwar, M. G.; Taylor, M. S. Halogen bonding in solution: thermodynamics and applications. Chem. Soc. Rev. 2013, 42, 1667-1680.

(56) Zhang, J.; Zhang, H.; Wu, T.; Wang, Q.; van der Spoel, D. Comparison of Implicit and Explicit Solvent Models for the Calculation of Solvation Free Energy in Organic Solvents. J. Chem. Theory Comput. 2017, 13, 1034-1043.

(57) Becke, A. D. Density-functional exchange-energy approximation with correct asymptotic-behavior. Phys. Rev. A: At., Mol., Opt. Phys. 1988, 38, 3098-3100.

(58) Perdew, J. P. Density-functional approximation for the correlation energy of the inhomogeneous electron gas. Phys. Rev. B: Condens. Matter Mater. Phys. 1986, 33, 8822-8824.

(59) Martins, S. A.; Sousa, S. F. Comparative Assessment of Computational Methods for the Determination of Solvation Free Energies in Alcohol-Based Molecules. J. Comput. Chem. 2013, 34, $1354-1362$.

(60) Zhang, H.; Jiang, Y.; Yan, H.; Cui, Z.; Yin, C. Comparative Assessment of Computational Methods for Free Energy Calculations of Ionic Hydration. J. Chem. Inf. Model. 2017, 57, 2763-2775.

(61) Lang, B. E. Solubility of Water in Octan-1-ol from (275 to 369) K. J. Chem. Eng. Data 2012, 57, 2221-2226.

(62) Marenich, A. V.; Jerome, S. V.; Cramer, C. J.; Truhlar, D. G. Charge Model 5: An Extension of Hirshfeld Population Analysis for the Accurate Description of Molecular Interactions in Gaseous and Condensed Phases. J. Chem. Theory Comput. 2012, 8, 527-541.

(63) Klamt, A. Conductor-like Screening Model for Real Solvents: A New Approach to the Quantitative Calculation of Solvation Phenomena. J. Phys. Chem. 1995, 99, 2224-2235.

(64) Klamt, A.; Jonas, V.; Bürger, T.; Lohrenz, J. C. W. Refinement and Parametrization of COSMO-RS. J. Phys. Chem. A 1998, 102, 50745085.

(65) Klamt, A. COSMO-RS from Quantum Chemistry to Fluid Phase Thermodynamics and Drug Design; Elsevier: Amsterdam, 2005.

(66) Klamt, A.; Schüürmann, G. COSMO: A New Approach to Dielectric Screening in Solvents with Explicit Expressions for the Screening Energy and its Gradient. J. Chem. Soc., Perkin Trans. 2 1993, 799-805.

(67) TURBOMOLE: Program Package for Ab Initio Electronic Structure Calculations; TURBOMOLE Gmbh: http://www.turbomole.com, 2017.

(68) Rappoport, D.; Furche, F. Property-optimized Gaussian basis sets for molecular response calculations. J. Chem. Phys. 2010, 133, 134105 .

(69) Eckert, F.; Klamt, A. COSMOtherm, version 18.0.0; COSMOlogic GmbH \& CoKG: Leverkusen, Germany, 2018.

(70) Klamt, A.; Eckert, F.; Reinisch, J.; Wichmann, K. Prediction of cyclohexane-water distribution coefficients with COSMO-RS on the SAMPL5 data set. J. Comput.-Aided Mol. Des. 2016, 30, 959-967.

(71) Kim, M. J.; Kwack, S. J.; Lim, S. K.; Kim, Y.J.; Roh, T. H.; Choi, S. M.; Kim, H. S.; Lee, B. M. Toxicological evaluation of isopropylparaben and isobutylparaben mixture in Sprague-Dawley rats following 28 days of dermal exposure. Regul. Toxicol. Pharmacol. 2015, 73, 544-551.

(72) Wang, J.; Wolf, R. M.; Caldwell, J. W.; Kollman, P. A.; Case, D. A. Development and Testing of a General AMBER Force Field. J. Comput. Chem. 2004, 25, 1157-1174.

(73) Singh, U. C.; Kollman, P. A. An Approach to Computing Electrostatic Charges for Molecules. J. Comput. Chem. 1984, 5, 129145.

(74) Besler, B. H.; Merz, K. M., Jr.; Kollman, P. A. Atomic Charges Derived from Semiempirical Methods. J. Comput. Chem. 1990, 11, 431-439.
(75) Hohenberg, P.; Kohn, W. Inhomogeneous Electron Gas. Phys. Rev. 1964, 136, B864-B871.

(76) Lee, C.; Yang, W.; Parr, R. G. Development of the Colle-Salvetti correlation-energy formula into a functional of the electron density. Phys. Rev. B: Condens. Matter Mater. Phys. 1988, 37, 785-789.

(77) Becke, A. D. Density-functional thermochemistry. III. The role of exact exchange. J. Chem. Phys. 1993, 98, 5648-5652.

(78) Kendall, R. A.; Dunning, T. H., Jr.; Harrison, R. J. Electron affinities of the first-row atoms revisited. Systematic basis sets and wave functions. J. Chem. Phys. 1992, 96, 6796-6806.

(79) Woon, D. E.; Dunning, T. H., Jr. Benchmark calculations with correlated molecular wave functions. I. Multireference configuration interaction calculations for the second row diatomic hydrides. J. Chem. Phys. 1993, 99, 1914-1929.

(80) Woon, D. E.; Dunning, T. H., Jr. Gaussian basis sets for use in correlated molecular calculations. III. The atoms aluminum through argon. J. Chem. Phys. 1993, 98, 1358-1371.

(81) Ghahremanpour, M. M.; van Maaren, P. J.; van der Spoel, D. Alexandria Library [Data Set]; Zenodo, 2017; http://doi.org/10.5281/ zenodo.1004711.

(82) Jorgensen, W. L.; Chandrasekhar, J.; Madura, J. D.; Impey, R. W.; Klein, M. L. Comparison of Simple Potential Functions for Simulating Liquid Water. J. Chem. Phys. 1983, 79, 926-935.

(83) Pronk, S.; Páll, S.; Schulz, R.; Larsson, P.; Bjelkmar, P.; Apostolov, R.; Shirts, M. R.; Smith, J. C.; Kasson, P. M.; van der Spoel, D.; Hess, B.; Lindahl, E. GROMACS 4.5: a high-throughput and highly parallel open source molecular simulation toolkit. Bioinformatics 2013, $29,845-854$

(84) Abraham, M. J.; Murtola, T.; Schulz, R.; Páll, S.; Smith, J. C.; Hess, B.; Lindahl, E. GROMACS: High performance molecular simulations through multi-level parallelism from laptops to supercomputers. SoftwareX 2015, 1-2, 19-25.

(85) Essmann, U.; Perera, L.; Berkowitz, M. L.; Darden, T.; Lee, H.; Pedersen, L. G. A Smooth Particle Mesh Ewald Method. J. Chem. Phys. 1995, 103, 8577-8593.

(86) Wennberg, C. L.; Murtola, T.; Hess, B.; Lindahl, E. LennardJones Lattice Summation in Bilayer Simulations Has Critical Effects on Surface Tension and Lipid Properties. J. Chem. Theory Comput. 2013, 9, $3527-3537$

(87) Caleman, C.; van Maaren, P. J.; Hong, M.; Hub, J. S.; Costa, L. T.; van der Spoel, D. Force Field Benchmark of Organic Liquids: Density, Enthalpy of Vaporization, Heat Capacities, Surface Tension, Isothermal Compressibility, Volumetric Expansion Coefficient, and Dielectric Constant. J. Chem. Theory Comput. 2012, 8, 61-74.

(88) Zubillaga, R. A.; Labastida, A.; Cruz, B.; Martínez, J. C.; Sánchez, E.; Alejandre, J. Surface Tension of Organic Liquids Using the OPLS/ AA Force Field. J. Chem. Theory Comput. 2013, 9, 1611-1615.

(89) Fischer, N. M.; van Maaren, P. J.; Ditz, J. C.; Yildirim, A.; van der Spoel, D. Properties of Organic Liquids when Simulated with LongRange Lennard-Jones Interactions. J. Chem. Theory Comput. 2015, 11, 2938-2944.

(90) Bashardanesh, Z.; van der Spoel, D. Impact of Dispersion Coefficient on Simulations of Proteins and Organic Liquids. J. Phys. Chem. B 2018, 122, 8018-8027.

(91) Hess, B.; Bekker, H.; Berendsen, H. J. C.; Fraaije, J. G. E. M. LINCS: A Linear Constraint Solver for Molecular Simulations. J. Comput. Chem. 1997, 18, 1463-1472.

(92) Bussi, G.; Donadio, D.; Parrinello, M. Canonical sampling through velocity rescaling. J. Chem. Phys. 2007, 126, 014101.

(93) Parrinello, M.; Rahman, A. Polymorphic Transitions in Single Crystals: A New Molecular Dynamics Method. J. Appl. Phys. 1981, 52, $7182-7190$

(94) Hickey, A. L.; Rowley, C. N. Benchmarking Quantum Chemical Methods for the Calculation of Molecular Dipole Moments and Polarizabilities. J. Phys. Chem. A 2014, 118, 3678-3687.

(95) Gutiérrez, I. S.; Lin, F.-Y.; Vanommeslaeghe, K.; Lemkul, J. A.; Armacost, K. A.; Brooks, C. L., III; MacKerell, A. D., Jr. Parametrization of halogen bonds in the CHARMM general force field: Improved 
treatment of ligand-protein interactions. Bioorg. Med. Chem. 2016, 24, $4812-4825$.

(96) Hidalgo, A.; Mora-Diez, N. Novel approach for predicting partition coefficients of linear perfluorinated compounds. Theor. Chem. Acc. 2016, 135, 18.

(97) Casoni, D.; Sârbu, C. The lipophilicity of parabens estimated on reverse phases chemically bonded and oil-impregnated plates and calculated using different computation methods. J. Sep. Sci. 2009, 32, 2377-2384.

(98) Kitagawa, S.; Li, H.; Sato, S. Skin Permeation of Parabens in Excised Guinea Pig Dorsal Skin, Its Modification by Penetration Enhancers and Their Relationship with n-Octanol/Water Partition Coefficients. Chem. Pharm. Bull. 1997, 45, 1354-1357.

(99) Kim, S.; Thiessen, P. A.; Bolton, E. E.; Chen, J.; Fu, G.; Gindulyte, A.; Han, L.; He, J.; He, S.; Shoemaker, B. A.; Wang, J.; Yu, B.; Zhang, J.; Bryant, S. H. PubChem substance and compound databases. Nucleic Acids Res. 2015, 44, D1202-D1213.

(100) Inoue, Y.; Hashizume, N.; Yakata, N.; Murakami, H.; Suzuki, Y.; Kikushima, E.; Otsuka, M. Unique physicochemical properties of perfluorinated compounds and their bioconcentration in common carp cyprinus carpio L. Arch. Environ. Contam. Toxicol. 2012, 62, 672-680. (101) Eadsforth, C. V.; Moser, P. Assessment of reverse-phase chromatographic methods for determining partition coefficients. Chemosphere 1983, 12, 1459-1475.

(102) Noble, A. Partition coefficients (n-octanol-water) for pesticides. J. Chromatogr. A 1993, 642, 3-14.

(103) Abraham, M. H.; Whiting, G. S.; Fuchs, R.; Chambers, E. J. Thermodynamics of solute transfer from water to hexadecane. J. Chem. Soc., Perkin Trans. 2 1990, 291-300.

(104) Zhang, J.; Tuguldur, B.; van der Spoel, D. Force Field Benchmark of Organic Liquids. 2. Gibbs Energy of Solvation. J. Chem. Inf. Model. 2015, 55, 1192-1201.

(105) Zhang, J.; Tuguldur, B.; van der Spoel, D. Correction to Force Field Benchmark of Organic Liquids. 2. Gibbs Energy of Solvation. J. Chem. Inf. Model. 2016, 56, 819-820.

(106) Kamaya, H.; Kaneshina, S.; Ueda, I. Partition equilibrium of inhalation anesthetics and alcohols between water and membranes of phospholipids with varying acyl chain-lengths. Biochim. Biophys. Acta, Biomembr. 1981, 646, 135-142.

(107) Duffy, E. M.; Jorgensen, W. L. Prediction of Properties from Simulations: Free Energies of Solvation in Hexadecane, Octanol, and Water. J. Am. Chem. Soc. 2000, 122, 2878-2888.

(108) Bannan, C. C.; Calabró, G.; Kyu, D. Y.; Mobley, D. L. Calculating Partition Coefficients of Small Molecules in Octanol/ Water and Cyclohexane/Water. J. Chem. Theory Comput. 2016, 12, 4015-4024.

(109) Benfenati, E.; Gini, G.; Piclin, N.; Roncaglioni, A.; Varia, M. R. Predicting $\log \mathrm{P}$ of pesticides using different software. Chemosphere 2003, 53, 1155-1164.

(110) Bordner, A. J.; Cavasotto, C. N.; Abagyan, R. A. Accurate Transferable Model for Water, n-Octanol, and n-Hexadecane Solvation Free Energies. J. Phys. Chem. B 2002, 106, 11009-11015.

(111) dos Reis, R. R.; Sampaio, S. C.; de Melo, E. B. The effect of different $\log \mathrm{P}$ algorithms on the modeling of the soil sorption coefficient of nonionic pesticides. Water Res. 2013, 47, 5751-5759.

(112) Geisler, A.; Oemisch, L.; Endo, S.; Goss, K.-U. Predicting Storage-Lipid Water Partitioning of Organic Solutes from Molecular Structure. Environ. Sci. Technol. 2015, 49, 5538-5545.

(113) Olguin, C. J. M.; Sampaio, S. C.; dos Reis, R. R. Statistical equivalence of prediction models of the soil sorption coefficient obtained using different log $\mathrm{P}$ algorithms. Chemosphere 2017, 184, 498-504.

(114) Mansouri, K.; Grulke, C. M.; Judson, R. S.; Williams, A. J. OPERA models for predicting physicochemical properties and environmental fate endpoints. J. Cheminf. 2018, 10, 10.

(115) Zhang, H.; Jiang, Y.; Yan, H.; Yin, C.; Tan, T.; van der Spoel, D. Free-Energy Calculations of Ionic Hydration Consistent with the Experimental Hydration Free Energy of the Proton. J. Phys. Chem. Lett. 2017, 8, 2705-2712.
(116) Zhang, H.; Yin, C.; Jiang, Y.; van der Spoel, D. Force Field Benchmark of Amino acids: I. Hydration and Diffusion in Different Water Models. J. Chem. Inf. Model. 2018, 58, 1037-1052. 